\title{
Disrupted functional connectivity between sub- regions in the sensorimotor areas and cortex in migraine without aura
}

\section{Zhaoxia Qin}

East China normal university

Jingjing Su

Shanghai Jiao Tong University School of Medicine

\section{Xin-Wei He}

Shanghai Jiao Tong University School of Medicine

\section{Shiyu Ban}

East China Normal University

\section{Qian Zhu}

East China Normal University

\section{Yangyang Cui}

East China Normal University

\section{Jilei Zhang}

Philips Healthcare

Yue Hu

Shanghai Jiao Tong University School of Medicine

Yi-Sheng Liu

Shanghai Jiao Tong University School of Medicine

Rong Zhao

Shanghai Jiao Tong University School of Medicine

\section{Yuan Qiao}

Shanghai Jiao Tong University School of Medicine

Jianqi Li

East china normal university

Jian-Ren Liu

Shanghai Jiao Tong University School of Medicine

Xiaoxia Du ( $\nabla$ xxdu@phy.ecnu.edu.cn )

East China Normal University https://orcid.org/0000-0001-8470-7852 
Keywords: Migraine, sensorimotor, sub-region, resting state, functional MRI, functional connectivity Posted Date: March 13th, 2020

DOI: https://doi.org/10.21203/rs.3.rs-17089/v1

License: (c) (1) This work is licensed under a Creative Commons Attribution 4.0 International License. Read Full License

Version of Record: A version of this preprint was published at The Journal of Headache and Pain on May 6th, 2020. See the published version at https://doi.org/10.1186/s10194-020-01118-1. 


\section{Abstract}

Background: Migraine is a severe and disabling brain disorder, and the exact neurological mechanisms remain unclear. Migraineurs have altered pain perception, and headache attacks disrupt their sensory information processing and sensorimotor integration. The altered functional connectivity (FC) of subregions of sensorimotor brain areas with other brain cortex associated with migraine needs further investigation.

Methods: Forty-eight migraine without aura (MWoAs) during the interictal phase and 48 age- and sexmatched healthy controls ( $\mathrm{HCs}$ ) underwent resting-state functional magnetic resonance imaging (fMRI) scans. We utilized seed-based functional connectivity analysis to investigate whether patients exhibited abnormal functional connectivity between sub-regions of sensorimotor brain areas and cortex regions.

Results : We found that MWoAs exhibited disrupted FC between the sensorimotor areas and the visual cortex, temporal cortex, posterior parietal lobule, prefrontal areas, precuneus, cingulate gyrus, sensorimotor areas proper and cerebellum areas compared with healthy controls. In addition, the clinical data of the patients, such as disease duration, pain intensity and HIT-6 score, were negatively correlated with these impaired FC s.

Conclusion : In MWoAs, the FCs between the sensorimotor brain areas and other brain regions was reduced. These disrupted FCs might contribute to abnormalities in visual processing, multisensory integration, nociception processing, spatial attention and intention and dysfunction in cognitive evaluation and modulation of pain. Recurrent headache attacks might lead to the disrupted network between L M1 (primary motor cortex) and temporal regions and between L S1 (primary somatosensory cortex) and temporal regions. Pain sensitivity and patient quality of life are closely tied to the abnormal functional connectivity between sensorimotor regions and other brain regions.

\section{Introduction}

Migraine is a severe and disabling brain disorder that is characterized by recurrent attacks of headache and multiple sensory symptoms[1, 2]. Multisensory integration of somatosensory, visual, auditory and olfactory stimuli by the migraine brain may be important for understanding migraine[3]. Migraineurs have altered pain perception, and headache attacks disrupt their sensory information processing and sensorimotor integration.

The mesial premotor cortex including pre-supplementary motor area (preSMA) and supplementary motor area proper (SMA), lateral premotor cortex including dorsal premotor cortex (PMd) and ventral premotor cortex (PMv), and primary sensorimotor cortex including (primary motor cortex (M1) and primary somatosensory cortex (S1) have been identified as key cortical areas for sensorimotor function [4]. The motor and premotor cortex (PMC) and S1 are involved in the spatial discrimination and pain intensity pathways[5]. Moreover, the motor areas, the PMC, the supplementary motor area (SMA) and the anterior cingulate cortex (ACC) constitute a module that contributes to response selection and the generation of 
sensory information[6]. Anatomically, first-order thalamus neurons located in the ventral posteromedial nucleus project mainly to the trigeminal areas of $\mathrm{S} 1$ as well as of the second somatosensory cortex (S2) and the insular cortices[7]. In addition, the S1, primary motor cortex (M1), and insular cortex have been implicated in the ascending trigemino-thalamo-cortical nociceptive pathway[8].

A previous study found functional abnormalities in the S1 and right PMC in patients[9]. Episodic migraineurs showed significant hypometabolism in the PMC and S1 relative to controls[10]. Connectivity between the left ventral striatum and ipsilateral preSMA was decreased in chronic migraine with medication-overuse headaches compared with chronic migraine alone[11]. A meta-analysis of excitatory primary motor cortex (M1) stimulation showed significant effects on reducing headache intensity and frequency of headache attacks in patients with migraine with a large effect size[12]. Migraine without aura showed decreased functional connectivity between the left hippocampus and contralateral SMA and bilateral inferior parietal gyri (IPG)[13]. Patients with a higher frequency of migraine attacks showed increased periaqueductal gray matter (PAG) connectivity with the $\mathrm{S} 1$ face representation area and the SMA, an area involved in pain expectancy[14].

The altered functional connectivity of sensorimotor regions with other brain areas would affect the multisensory integration and perception and processing of pain, and the intrinsic FC between sub-regions of sensorimotor areas and the cortex associated with migraine without aura needs further investigation. We speculate that the interactions between sensorimotor regions and multiple cortical areas are necessary for the integration of information within and across the sensory modalities and, thus, could play an important role in the initiation of migraine attack and/or the development of its associated symptoms. Mayka et al. developed the Human Motor Area Template (HMAT), which used outcomes from ALE analysis in combination with previously suggested anatomical guidelines and can be used for seedbased functional connectivity analysis[4]. In this study, we tested the following hypotheses. 1) The intrinsic FCs of the sub-regions of sensorimotor areas to many important brain regions in patients with migraine without aura (MWoA) may be altered. 2) These altered sensorimotor region connectivity patterns may be associated with features of disease severity, such as disease duration, pain intensity, attack frequency, and the Migraine Disability Assessment Scale (MIDAS) and Headache Impact Test (HIT6) scores.

\section{Methods}

\section{Subjects}

Forty-eight patients with MWoA (mean \pm SD age $=38.1 \pm 10.4$ years) and 48 healthy controls (HCs) (mean \pm SD age $=39.0 \pm 11.0$ years) were enrolled from the outpatient clinic of the Department of Neurology at Shanghai Ninth People's Hospital. Patients were diagnosed with MWoA by a neurologist based on the International Classification of Headache Disorders 3rd edition criteria[2]. Demographic and clinical data were registered and evaluated in our headache database, including age, sex, disease duration, attack frequency (times/month), attack duration (hours) and pain intensity of migraine attacks assessed by a 
visual analogue scale (VAS)[15]. Patients also completed the MIDAS[16] and HIT-6[17] regarding the accurate assessment of their headache-related disability. All patients with MWoA were scanned during an interictal period, with no headache 48 hours before or 24 hours after MRI scans, and they did not report a migraine attack or discomfort during the MRI scans. Migraineurs reported that they did not take preventive medication and did not suffer chronic migraine. The HCs had no headaches or chronic pain disorders in the past year. Moreover, the immediate family members of the HCs did not suffer from migraine or other headaches. Other exclusion criteria were the following: left-handedness, drug abuse, any neurological or psychiatric diseases, metabolic diseases (e.g., diabetes mellitus) or cardiovascular diseases based on clinical examination and structured interviews. The demographic and clinical data are provided in Table 1.

Table 1

Demographic data and clinical scores of the MWoA group and control group.

\begin{tabular}{|llll|}
\hline & Migraine group & Control group & \\
\hline $\mathrm{N}$ & $($ Mean \pm SD) & $($ Mean \pm SD) & P value \\
\hline Sex (male) & 48 & 48 & 1 \\
\hline Age (years) & $29.2 \%$ & $29.2 \%$ & 1 \\
\hline Disease duration (years) & $38.1 \pm 10.4$ & $39.0 \pm 11.0$ & 0.68 \\
\hline Attack duration (hours) & $8.5 \pm 6.0$ & - & - \\
\hline Attack frequency (times/months) & $15.3 \pm 18.4$ & - & - \\
\hline Pain intensity VAS score & $3.8 \pm 3.3$ & - & - \\
\hline MIDAS score & $7.2 \pm 1.8$ & - & - \\
\hline HIT-6 score & $23.1 \pm 28.6$ & - & - \\
\hline $\begin{array}{l}\text { VAS: visual analogue scale, MIDAS: migraine disability assessment scale, HIT-6: headache impact } \\
\text { test, }-: \text { No data }\end{array}$ & $60.4 \pm 12.0$ & & - \\
\hline
\end{tabular}


Table 2

Brain regions with decreased functional connectivity $(\mathrm{FC})$ in migraine without aura (MWoA) compared to healthy controls $(\mathrm{HC})$.

\section{Predominant regions in the cluster}

Seed in L M1 (MWoA group < Control group)

Right middle occipital gyrus

Right middle temporal gyrus

Right superior temporal gyrus

Parietal_Sup_L (aal)

Right postcentral gyrus

Right middle frontal gyrus

Seed in R M1 (MWoA group < Control group)

Right middle occipital gyrus

Right superior temporal gyrus

Parietal_Sup_L (aal)

Postcentral_R (aal)

Right cuneus

Seed in L S1 (MWoA group < Control group)

Precuneus_L (aal) extending to

Bilateral postcentral gyrus
Cluster size

Peak T

\section{MNI coordinates}

$$
\begin{array}{lll}
x & y & z
\end{array}
$$

\begin{tabular}{llllll}
199 & -5.28 & 36 & -69 & 3 & 0.000 \\
151 & -5.19 & 57 & -57 & 0 & 0.000 \\
177 & -4.85 & 63 & -27 & 15 & 0.000 \\
303 & -4.73 & -30 & -48 & 57 & 0.000 \\
342 & -4.51 & 30 & -45 & 66 & 0.000 \\
135 & -4.44 & 36 & -3 & 48 & 0.001 \\
\hline 122 & -5.00 & 36 & -69 & 6 & 0.002 \\
60 & -4.59 & 63 & -27 & 15 & 0.046 \\
307 & -4.57 & -30 & -45 & 57 & 0.000 \\
164 & -4.24 & 33 & -42 & 57 & 0.000 \\
64 & -3.97 & 21 & -93 & 18 & 0.036
\end{tabular}

$176(971)$

$-5.73$

$-9$

$-48$

69

0.000
Bilateral Paracentral lobule

Precuneus_R (aal)

Right middle temporal gyrus

Right superior temporal gyrus

Left inferior parietal lobule

Left middle temporal gyrus

Right inferior frontal gyrus

210 (971)

112 (971)

$112(971)$

$\begin{array}{llllll}144 & -5.25 & 57 & -54 & 0 & 0.001 \\ 195 & -4.96 & 63 & -30 & 15 & 0.000 \\ 157 & -4.84 & -57 & -39 & 21 & 0.000 \\ 108 & -4.58 & -45 & -66 & 12 & 0.004 \\ 61 & -4.54 & 48 & 33 & 9 & 0.047\end{array}$

The results were assigned thresholds at $p<0.001$ (voxel level) and FWE corrected to $p<0.05$ at the cluster level. 


\begin{tabular}{|c|c|c|c|c|c|c|}
\hline \multirow{2}{*}{$\begin{array}{l}\text { Predominant regions in the cluster } \\
\text { Postcentral_L (aal) }\end{array}$} & \multirow{2}{*}{$\begin{array}{l}\text { Cluster size } \\
76\end{array}$} & \multirow{2}{*}{$\begin{array}{l}\text { Peak T } \\
-4.22\end{array}$} & \multicolumn{3}{|c|}{ MNI coordinates } & \multirow[b]{2}{*}{0.020} \\
\hline & & & -21 & -30 & 66 & \\
\hline Right middle frontal gyrus & 112 & -4.22 & 45 & 0 & 45 & 0.003 \\
\hline \multicolumn{7}{|l|}{ Seed in R S1 (MWoA group < Control group) } \\
\hline Precuneus_R (aal) extending to & $166(1219)$ & -5.13 & 12 & -42 & 63 & 0.000 \\
\hline Precuneus_L (aal) & 98 (1219) & & & & & \\
\hline Right cingulate gyrus & 67 (1219) & & & & & \\
\hline Bilateral postcentral gyrus & $320(1219)$ & & & & & \\
\hline Bilateral paracentral lobule & $252(1219)$ & & & & & \\
\hline Bilateral precentral gyrus & $246(1219)$ & & & & & \\
\hline Right superior temporal gyrus & 86 & -4.78 & 63 & -30 & 15 & 0.011 \\
\hline Left inferior parietal lobe & 61 & -4.59 & -60 & -42 & 21 & 0.046 \\
\hline Occipital_Sup_L (aal) & 87 & -4.13 & -21 & -60 & 18 & 0.010 \\
\hline \multicolumn{7}{|l|}{ Seed in L SMA (MWoA Group < HC Group) } \\
\hline Precentral_L (aal) extending to & $95(665)$ & -5.62 & -51 & 0 & 42 & 0.000 \\
\hline Left precuneus & $112(665)$ & & & & & \\
\hline Parietal_Inf_L (aal) & $210(665)$ & & & & & \\
\hline Postcentral_L (aal) & $198(665)$ & & & & & \\
\hline Right middle frontal gyrus extending to & $131(852)$ & -5.55 & 36 & -6 & 51 & 0.000 \\
\hline Right inferior parietal lobule & $145(852)$ & & & & & \\
\hline Postcentral_R (aal) & $274(852)$ & & & & & \\
\hline Precentral_R (aal) & $177(852)$ & & & & & \\
\hline Right cuneus & $217(605)$ & -5.22 & 15 & -78 & 30 & 0.000 \\
\hline Left middle temporal gyrus & 77 (605) & & & & & \\
\hline Right superior temporal gyrus & 76 & -4.95 & 63 & -27 & 15 & 0.020 \\
\hline Right middle temporal gyrus & 267 & -4.72 & 60 & -54 & 6 & 0.000 \\
\hline Seed in R SMA (MWoA Group $<$ HC Grou & & & & & & \\
\hline
\end{tabular}

The results were assigned thresholds at $p<0.001$ (voxel level) and FWE corrected to $p<0.05$ at the cluster level. 


\begin{tabular}{|c|c|c|c|c|c|c|}
\hline \multirow{2}{*}{$\begin{array}{l}\text { Predominant regions in the cluster } \\
\text { Right middle frontal gyrus extending to }\end{array}$} & \multirow{2}{*}{$\begin{array}{l}\text { Cluster size } \\
104(1942)\end{array}$} & \multirow{2}{*}{$\begin{array}{l}\text { Peak T } \\
-5.57\end{array}$} & \multicolumn{3}{|c|}{ MNI coordinates } & \multirow[b]{2}{*}{0.000} \\
\hline & & & 39 & -6 & 51 & \\
\hline Bilateral postcentral gyrus & $373(1942)$ & & & & & \\
\hline Bilateral precentral Gyrus & $330(1942)$ & & & & & \\
\hline Bilateral inferior parietal lobule & $243(1942)$ & & & & & \\
\hline Bilateral cuneus & $232(1942)$ & & & & & \\
\hline Bilateral precuneus & $221(1942)$ & & & & & \\
\hline Right middle temporal gyrus & 91 & -4.28 & 57 & -54 & -6 & 0.009 \\
\hline \multicolumn{7}{|c|}{ Seed in R preSMA (MWoA Group $<$ HC Group) } \\
\hline Left precuneus & 110 & 4.05 & -18 & -72 & 39 & 0.004 \\
\hline \multicolumn{7}{|l|}{ Seed in R PMd (MWoA Group < HC Group) } \\
\hline Left precuneus & 306 & -4.88 & -18 & -57 & 54 & 0.000 \\
\hline Left middle occipital gyrus & 200 & -4.75 & -36 & -78 & 9 & 0.000 \\
\hline Cerebellum_8_R & 83 & -4.49 & 18 & -60 & -57 & 0.013 \\
\hline Right cuneus & 144 & -4.47 & 21 & -93 & 18 & 0.001 \\
\hline Right middle occipital gyrus & 103 & -4.11 & 36 & -81 & 0 & 0.005 \\
\hline Right postcentral gyrus & 101 & -4.11 & 9 & -45 & 66 & 0.005 \\
\hline Left cuneus & 68 & -3.84 & -18 & -87 & 18 & 0.031 \\
\hline \multicolumn{7}{|l|}{ Seed in L PMv (MWoA Group < HC Group) } \\
\hline Left precentral gyrus & 107 & -4.50 & -27 & -30 & 57 & 0.004 \\
\hline Right cingulate gyrus & 111 & -3.92 & -9 & -9 & 39 & 0.003 \\
\hline Right postcentral gyrus & 75 & -3.77 & 12 & -45 & 69 & 0.022 \\
\hline \multicolumn{7}{|l|}{ Seed in R PMv (MWoA Group < HC Group) } \\
\hline Bilateral precuneus extending to & $139(469)$ & -4.75 & 12 & -45 & 63 & 0.000 \\
\hline Bilateral postcentral gyrus & $173(469)$ & & & & & \\
\hline Supp_Motor_Area_L (aal) & 109 & -4.32 & 0 & -18 & 51 & 0.004 \\
\hline
\end{tabular}




\section{Mri Acquisition}

MRI was carried out in a 3.0 T Siemens Trio Tim system with a 12-channel head coil at the Shanghai Key Laboratory of Magnetic Resonance (East China Normal University, Shanghai, China). All subjects' head movements were minimized with a Siemens dedicated filler. T1-weighted anatomical images were collected using a 3-dimensional magnetization-prepared rapid-acquisition gradient-echo pulse sequence (repetition time $=2530 \mathrm{~ms}$, echo time $=2.34 \mathrm{~ms}$, inversion time $=1100 \mathrm{~ms}$, flip angle $=7^{\circ}$, number of slices $=192$, sagittal orientation, field of view $=256 \times 256 \mathrm{~mm}^{2}$, matrix size $=256 \times 256$, slice thickness $=$ $1 \mathrm{~mm}, 50 \%$ gap). Following this, subjects were asked to remain motionless, keep their eyes closed, and stay awake and relaxed. Resting-state images consisted of 210 volumes of a T2*-weighted gradient-echo echo planar imaging pulse sequence (33 slices of $3.5 \mathrm{~mm}$ thickness, repetition time $=2000 \mathrm{~ms}$, echo time $=30 \mathrm{~ms}$, flip angle $=90^{\circ}$, transverse orientation, field of view $=220 \times 220 \mathrm{~mm}^{2}$, matrix size $=64 \times 64,25 \%$ distance factor).

\section{Data Preprocessing}

Functional data were preprocessed using the Data Processing Assistant for Resting-State fMRI (DPARSF, http://rfmri.org/DPARSF) and analyzed with Statistical Parametric Mapping (SPM12, http://www.fil.ion.ucl.ac.uk/spm). To avoid scanner instability, the first 10 volumes were discarded. The images for each subject were motion-corrected, and in no subjects was there significant movement (> $2 \mathrm{~mm}$ in any direction). The data from all the subjects were used for the subsequent analysis. We utilized the Friston[18] 24-parameter model to regress out the head motion effects from realignment. Functional volumes were slice-timing corrected and coregistered to the individual structural images without resampling. The transformed structural images were segmented [19] and normalized to the Montreal Neurological Institute (MNI) space. Based on the same deformation field, functional images were then normalized to the MNI space $\left(3 \times 3 \times 3 \mathrm{~mm}^{3}\right)$ using the Diffeomorphic Anatomical Registration Through Exponentiated Lie Algebra (DARTEL) tool [20]. Spatial smoothing was performed on the functional images by a $6 \mathrm{~mm}$ FWHM Gaussian filter. We performed temporal bandpass filtering $(0.01<f<0.1 \mathrm{~Hz})$, and the mean signals from the white matter, cerebrospinal fluid, and linear trends were also included as covariates to reduce the influence of low-frequency drift and high-frequency respiratory and cardiac effects.

\section{Seed-based Fc}

We calculated Pearson's correlation coefficients of the mean time courses extracted from the HMAT ROIs with the signals of whole-brain voxels. The HMAT ROls included M1, S1, the SMA, the preSMA, the PMd, and the PMv. To improve the normality of the data distribution, we converted FC maps to FC Z-value maps by Fisher's Z-transformation. Then, the voxelwise two-sample t-test with sex and age as covariates was conducted within a brain mask. The two-tailed (voxel-level) threshold was $p<0.001$, which was 
familywise error (FWE) corrected to $p<0.05$ (cluster level). Surviving clusters were deemed significant. Individual mean z-values for the surviving clusters were extracted to determine Spearman's correlation correlations with clinical data features, including disease duration, attack frequency, and attack duration, as well as VAS, MIDAS and HIT-6 scores. Thresholds were $p<0.05$, one-tailed, and FDR corrected for multiple comparisons.

\section{Results}

\section{Demographic and clinical characteristics}

The demographic and clinical data of the migraine and control groups are presented in Table 1. The ages and gender proportions did not differ between the two groups.

\section{Seed-based FC}

1. The patients with MWoAs showed weaker FCs between L M1 and the right middle occipital gyrus, right middle temporal gyrus, right superior temporal gyrus, left superior parietal gyrus, right postcentral gyrus, and right middle frontal gyrus (Figure 1). We also found decreased FCs between $\mathrm{R}$ M1 and the right middle occipital gyrus, right superior temporal gyrus, left superior parietal gyrus, right postcentral gyrus, and right cuneus in patients with MWoAs relative to HC (Figure 1).

2. The patients with MWoAs showed weaker FCs between L S1 and the bilateral precuneus, bilateral postcentral gyrus, bilateral paracentral lobule, bilateral middle temporal gyrus, right superior temporal gyrus, left inferior parietal lobule and right inferior frontal gyrus and right middle frontal gyrus (Figure 1). We also found decreased FCs between R S1 and the bilateral precuneus, right cingulate gyrus, bilateral postcentral gyrus, bilateral paracentral lobule, bilateral precentral gyrus, right superior temporal gyrus, left inferior parietal lobe, and left occipital superior gyrus (Figure 1).

3. The patients with MWoAs showed weaker FCs between the L SMA and bilateral precentral gyrus, bilateral postcentral gyrus, left precuneus and bilateral inferior parietal lobule and right middle frontal gyrus, right cuneus, bilateral middle temporal gyrus, and right superior temporal gyrus (Figure 2). The patients with MWoAs showed weaker FCs between the R SMA and the right middle frontal gyrus, bilateral postcentral gyrus, bilateral inferior parietal lobule, bilateral cuneus, bilateral precuneus, and right middle temporal gyrus (Figure 2).

4. The patients with MWoAs showed weaker FCs between the L PMv and the left precentral gyrus, right cingulate gyrus, and right postcentral gyrus (Figure 2). The patients with MWoAs showed weaker FCs between the R PMv and the bilateral precuneus, bilateral postcentral gyrus and L SMA (Figure 2).

5. The patients with MWoAs showed a weaker FC between the R preSMA and the left precuneus (Figure $3)$.

6. The patients with MWoAs showed weaker FCs between the R PMd and the left precuneus, bilateral middle occipital gyrus, cerebellum areas, bilateral cuneus, and right postcentral gyrus (Figure 4). 


\section{Correlations with clinical variables in migraine}

A negative correlation was found between the disease duration of the patients and the FC Z scores between the L M1 and the R middle temporal gyrus (MTG). The pain intensity of the patients was negatively correlated with the FC Z scores between the L SI and the R superior temporal gyrus (STG). The FC Z scores between the L S1 and the R MTG and R STG were also negatively correlated with disease duration in MWoAs. The HIT-6 score in MWoAs was negatively correlated with the FC Z score between the $\mathrm{L} P M v$ and the $\mathrm{R}$ cingulate gyrus (Figure 5).

\section{Discussion}

Our previous study found that MWoAs exhibited functional abnormalities in the S1 and PMC and weaker FCs between the $\mathrm{S} 1$ and brain areas within the pain intensity and spatial discrimination pathways and the trigemino-thalamo-cortical nociceptive pathway[21]. Consistent with a previous study, we found disrupted functional connectivity between $\mathrm{S} 1$ and brain regions involved in pain processing. In addition, we subdivided the sensorimotor areas as preSMA, SMA, PMd, PMv, M1, and S1 and found disrupted FCs between these sensorimotor brain regions and numerous other regions of the brain, such as the visual cortex, temporal cortex, posterior parietal lobule, prefrontal areas, precuneus, cingulate gyrus, sensorimotor areas proper and cerebellum areas. These abnormal interactions between sensorimotor regions and multiple brain areas might contribute to dysfunction of the integration of information within and across the sensory modalities and, thus, could lead to the initiation of migraine attack and/or its associated symptoms. These impaired FCs were negatively correlated with clinical data, such as disease duration, pain intensity, and HIT-6 scores, which provided increased evidence that impaired FC between sub-regions of sensorimotor regions and multiple brain areas is involved in the pathophysiological mechanism of migraine.

$\mathrm{M} 1$ is the main contributor to generating neural impulses that pass down to the spinal cord and control the execution of movement[22] and has been implicated in the ascending trigemino-thalamo-cortical nociceptive pathway [8]. Activation of S1 in studies of laser-evoked pain supports a role for S1 in the sensory aspects of pain, including discrimination and localization of pain intensity[23]. The SMA has many proposed functions, including the internally generated planning of movement, the planning of sequences of movement, and the coordination of the two sides of the body, such as in bi-manual coordination [24, 25]. The SMA is involved in pain anticipation[26]. The activity of pre-SMA neurons suggests that it involves a mechanism for switching from automatic to controlled eye movements[27]. The PMd may participate in learning to associate arbitrary sensory stimuli with specific movements or learning arbitrary response rules[28,29]. The PMv is often studied with respect to its role in the sensory guidance of movement. Neurons here are responsive to tactile stimuli, visual stimuli, and auditory stimuli [30-32].

We found decreased functional connectivity between M1 and many other brain areas, such as the right middle occipital gyrus, right cuneus, right middle and superior temporal gyrus, left superior parietal gyrus, 
right postcentral gyrus, and right middle frontal gyrus, which are involved in pain perception and pain processing. A meta-analysis showed that excitatory M1 stimulation had a significant effect on reducing headache intensity and frequency of headache attacks in patients with migraine with a large effect size[12]. The middle occipital gyrus and right cuneus are involved in visual processing, and the migraineurs showed significantly higher activation than the control group in the left cuneus while viewing negative pictures[33]. Moreover, a systematic review indicated that occipital nerve stimulation can significantly reduce the pain intensity and the number of days with headache in patients with migraine[34]. The temporal pole participates in pain processing by mediating affective responses to painful stimuli and by acting as a multisensory integration zone responsible for processing painful, visual, auditory, and olfactory stimuli $[3,35,36]$. The posterior parietal lobule is now believed to underpin higher-order processes of sensory inputs, multisensory and sensorimotor integration, spatial attention, intention, and the conjoint representation of external space and the body[37]. The somatosensory cortex is contained in the postcentral gyrus, and a previous study found functional abnormalities in S1 and disrupted functional connectivity between S1 and other cortical regions[9]. The MFG, as part of the prefrontal cortex, is thought to be involved in the cognitive evaluation and modulation of pain[38]. The frequency of migraine attacks and the duration of the disorder had a significant impact on cortical thickness in the sensorimotor cortex and the middle frontal gyrus[39]. Disrupted FC between M1 and these brain regions might contribute to visual processing, multisensory integration, spatial attention and intention abnormalities, and dysfunction in the cognitive evaluation and modulation of pain.

We found decreased functional connectivity between S1, the SMA and many other brain areas, such as the bilateral precuneus, bilateral postcentral gyrus, bilateral paracentral lobule, bilateral precentral gyrus, right cingulate gyrus, bilateral middle temporal gyrus, right superior temporal gyrus, bilateral inferior parietal lobule, right inferior and middle frontal gyrus, and left occipital superior gyrus and right cuneus. In addition, patients with MWoAs showed a weaker FC between the R preSMA and the left precuneus. The precuneus has been proposed to participate in information transfer and multimodal integration, which might be essential for the processing of spontaneous thoughts and for internal awareness[40]. The postcentral gyrus, paracentral lobule, and precentral gyrus are involved in sensorimotor networks, and a previous study found functional abnormalities and abnormal cortical thickness or gray matter volume in the sensorimotor network in migraineurs $[9,41,42]$. Gray matter volume decreases in the right anterior cingulate are related to the estimated frequency of headache attacks[43]. The temporal pole has been demonstrated to participate in pain processing and multisensory integration $[3,35,36]$. The inferior parietal lobule has been demonstrated to be involved in spatial discrimination and attention to pain [4446]. The prefrontal region is thought to be involved in the cognitive evaluation and modulation of pain[38]. The middle occipital gyrus and right cuneus are involved in visual processing, and significantly increased cortical thickness was found in the lateral occipital cortex[47].

The bilateral PMCs were activated during a delayed match-to-sample task using thermal stimuli in healthy people; they receive input information from the ACC and contribute to the communication and selection of a decision about the nature of the afferent sensory information during both intensity and spatial discrimination[5]. The patients with MWoAs showed weaker FCs between R PMd and the left 
precuneus, bilateral middle occipital gyrus, cerebellum regions, bilateral cuneus, and right postcentral gyrus. The patients with MWoAs showed weaker FCs between the PMv and left precentral gyrus, right cingulate gyrus, bilateral precuneus, bilateral postcentral gyrus and L SMA. Numerous regions mentioned above are involved in the pathology of migraine. In addition, a previous study found structural changes in cerebellum-associated migraine[48]. The cerebellum has been demonstrated to be involved in human nociception[49] and is even suggested to play a modulating role in pain perception[50].

The disease duration of the patients was negatively correlated with the FC Z scores between the L M1 and the R MTG and R STG and the FC Z scores between the L S1 and the R MTG and R STG, which suggests that recurrent headache attacks may drive functional changes and contribute to this disrupted network among the L M1, L S1 and temporal regions. The pain intensity of the patients was negatively correlated with the FC Z scores between the L SI and R STG. This result further indicates that the reduced FC between the $L S 1$ and R STG is indeed a maladaptive functional plasticity closely related to pain intensity in MWoAs. The HIT-6 score in MWoAs was negatively correlated with the FC Z score between the $\mathrm{L} P M v$ and $\mathrm{R}$ cingulate gyrus, which suggests that the reduced $\mathrm{FC}$ is significantly related to the impact of recurrent migraine attacks on daily life.

\section{Limitation}

Although our research revealed that MWoAs exhibited deficits in the functional connectivity between sensorimotor regions and other cortical areas, the current study had several limitations. First, we examined patients only in the interictal phase, and therefore, functional connectivity in the ictal phase must also be explored. Second, we focused on MWoAs but did not examine migraineurs with aura and chronic migraineurs. In a future study, we will evaluate the functional abnormalities in the sensorimotor regions of these other two populations during the ictal phase.

\section{Conclusion}

We found that MWoAs exhibited disrupted functional connectivity between the sensorimotor areas and the visual cortex, temporal cortex, posterior parietal lobule, prefrontal areas, precuneus, cingulate gyrus, sensorimotor areas proper and cerebellum areas compared with healthy controls. These disrupted FCs might contribute to abnormalities in visual processing, multisensory integration, nociception processing, spatial attention and intention and dysfunction in cognitive evaluation and modulation of pain. Recurrent headache attacks might lead to the disrupted network between the L M1 and temporal regions and between the L S1 and temporal regions. Pain sensitivity and patient quality of life are closely tied to abnormal functional connectivity between sensorimotor regions and other brain areas.

\section{Abbreviations}

ACC: anterior cingulate cortex; fMRI: functional magnetic resonance imaging; GM: Gray matter; HCs: Healthy controls; FC: functional connectivity (FC); FWE: Familywise error; FDR: false discovery rate; 
HMAT: Human Motor Area Template; HIT-6: Headache Impact Test; IPG: inferior parietal gyri; MTG: middle temporal lobule; MWoAs: migraineurs without aura; MIDAS: Migraine Disability Assessment Scale; MNI: Montreal Neurological Institute; MRI: magnetic resonance imaging; M1 : primary motor cortex; PAG: periaqueductal gray matter; PMC: premotor cortex; preSMA: pre-supplementary motor area; PMv : ventral premotor cortex; PMd : dorsal premotor cortex; SMA : supplementary motor cortex; S1: primary somatosensory cortex; S2: second somatosensory cortex; STG: superior temporal gyrus;

\section{Declarations}

\section{Funding}

This research was supported by grants from the National Natural Science Foundation of China (Nos. 81571658 to X. X. Du), the National Natural Science Foundation of China (81271302 to J.R. Liu), the Research Innovation Project from Shanghai Municipal Science and Technology Commission (No. 14JC1404300, to J.R. Liu), the "Prevention and Control of Chronic Diseases Project" of Shanghai Hospital Development Center (No. SHDC12015310, to J.R. Liu), a project from SHSMU-ION Research Center for Brain Disorders (No. 2015NKX006, to J.R. Liu), a project from Shanghai Municipal Education Commission-Gaofeng Clinical Medicine Grant Support (No. 20161422 to J. R. Liu), a Clinical Research Project from Shanghai Jiao Tong University School of Medicine (No. DLY201614 to J.R. Liu), and the Biomedicine Key Program from Shanghai Municipal Science and Technology Commission (No. 16411953100 to J.R. Liu).

\section{Competing interests}

The authors declare no potential conflicts of interest with respect to the research, authorship, and/or publication of this article.

\section{Ethics approval and consent to participate}

All procedures were approved by the East China Normal University Committee on Human Research (Project No. HR2015/0311) and by the independent Ethics Committee of Shanghai Ninth People's Hospital (Project No [2016]01). The study conformed to the ethical principles stated in the Declaration of Helsinki. Participants received a complete description about the experiment and gave written informed consent.

\section{Consent for Publication}

Not applicable

\section{Authors' contributions}

$X D, J L$ and ZQ designed the study. JS XH, ZQ, JZ, SB, QZ, YC, YH, YL, RZ, and YQ, performed the experiments. ZQ, JZ, XH and JS analyzed the data. 
$X D, J L$ and ZQ prepared the manuscript. All authors read and approved the final manuscript.

Availability of data and materials

The datasets generated during and/or analyzed during the current study are available from the corresponding author on reasonable request.

\section{Acknowledgments}

We thank the patients who participated in the study for their support.

\section{References}

1. Goadsby PJ, Holland PR, Martins-Oliveira M, Hoffmann J, Schankin C, Akerman S: Pathophysiology of migraine: a disorder of sensory processing. Physiological reviews 2017, 97(2):553-622.

2. Headache: Classification Committee of the International Headache Society (IHS) The International Classification of Headache Disorders, 3rd edition. Cephalalgia 2018, 38(1):1-211.

3. Schwedt TJ: Multisensory integration in migraine. Current opinion in neurology 2013, 26(3):248.

4. Mayka MA, Corcos DM, Leurgans SE, Vaillancourt DE: Three-dimensional locations and boundaries of motor and premotor cortices as defined by functional brain imaging: a meta-analysis. Neurolmage 2006, 31(4):1453-1474.

5. Oshiro Y, Quevedo AS, McHaffie JG, Kraft RA, Coghill RC: Brain mechanisms supporting discrimination of sensory features of pain: a new model. Journal of Neuroscience 2009, 29(47):14924-14931.

6. Cavina-Pratesi C, Valyear KF, Culham JC, Köhler S, Obhi SS, Marzi CA, Goodale MA: Dissociating arbitrary stimulus-response mapping from movement planning during preparatory period: evidence from event-related functional magnetic resonance imaging. Journal of Neuroscience 2006, 26(10):2704-2713.

7. Noseda R, Jakubowski M, Kainz V, Borsook D, Burstein R: Cortical projections of functionally identified thalamic trigeminovascular neurons: implications for migraine headache and its associated symptoms. Journal of Neuroscience 2011, 31(40):14204-14217.

8. Burstein R, Noseda R, Borsook D: Migraine: multiple processes, complex pathophysiology. Journal of Neuroscience 2015, 35(17):6619-6629.

9. Zhang J, Su J, Wang M, Zhao Y, Zhang QT, Yao Q, Lu H, Zhang H, Li GF, Wu YL et al: The sensorimotor network dysfunction in migraineurs without aura: a resting-state fMRI study. Journal of neurology 2017, 264(4):654-663.

10. Kim JH, Kim S, Suh SI, Koh SB, Park KW, Oh K: Interictal metabolic changes in episodic migraine: a voxel-based FDG-PET study. Cephalalgia 2009:no-no.

11. Androulakis XM, Rorden C, Peterlin BL, Krebs K: Modulation of salience network intranetwork resting state functional connectivity in women with chronic migraine. Cephalalgia 2018, 38(11):1731-1741. 
12. Feng Y, Zhang B, Zhang J, Yin Y: Effects of Non-invasive Brain Stimulation on Headache Intensity and Frequency of Headache Attacks in Patients With Migraine: A Systematic Review and MetaAnalysis. Headache: The Journal of Head and Face Pain 2019, 59(9):1436-1447.

13. Wei H-L, Chen J, Chen Y-C, Yu Y-S, Zhou G-P, Qu L-J, Yin X, Li J, Zhang H: Impaired functional connectivity of limbic system in migraine without aura. Brain imaging and behavior 2019:1-10.

14. Solstrand Dahlberg L, Linnman CN, Lee D, Burstein R, Becerra L, Borsook D: Responsivity of periaqueductal gray connectivity is related to headache frequency in episodic migraine. Frontiers in neurology 2018, 9:61.

15. Hawker GA, Mian S, Kendzerska T, French M: Measures of adult pain: Visual analog scale for pain (vas pain), numeric rating scale for pain (nrs pain), mcgill pain questionnaire (mpq), short-form mcgill pain questionnaire (sf-mpq), chronic pain grade scale (cpgs), short form-36 bodily pain scale (sf-36 bps), and measure of intermittent and constant osteoarthritis pain (icoap). Arthritis care \& research 2011, 63(S11):S240-S252.

16. Stewart WF, Lipton RB, Whyte J, Dowson A, Kolodner K, Liberman Ja, Sawyer J: An international study to assess reliability of the Migraine Disability Assessment (MIDAS) score. Neurology 1999, 53(5):988-988.

17. Kosinski M, Bayliss M, Bjorner J, Ware J, Garber W, Batenhorst A, Cady R, Dahlöf C, Dowson A, Tepper S: A six-item short-form survey for measuring headache impact: The HIT-6 ${ }^{\mathrm{m}}$. Quality of Life Research 2003, 12(8):963-974.

18. Friston KJ, Williams S, Howard R, Frackowiak RS, Turner R: Movement-related effects in fMRI timeseries. Magnetic resonance in medicine 1996, 35(3):346-355.

19. Ashburner J, Friston KJ: Unified segmentation. Neurolmage 2005, 26(3):839-851.

20. Ashburner J: A fast diffeomorphic image registration algorithm. Neurolmage 2007, 38(1):95-113.

21. Zhang J, Su J, Wang M, Zhao Y, Zhang Q-T, Yao Q, Lu H, Zhang H, Li G-F, Wu Y-L: The sensorimotor network dysfunction in migraineurs without aura: a resting-state fMRI study. Journal of neurology 2017, 264(4):654-663.

22. Rivara CB, Sherwood CC, Bouras C, Hof PR: Stereologic characterization and spatial distribution patterns of Betz cells in the human primary motor cortex. The Anatomical Record Part A: Discoveries in Molecular, Cellular, and Evolutionary Biology: An Official Publication of the American Association of Anatomists 2003, 270(2):137-151.

23. Valentini E, Hu L, Chakrabarti B, Hu Y, Aglioti SM, lannetti GD: The primary somatosensory cortex largely contributes to the early part of the cortical response elicited by nociceptive stimuli. Neurolmage 2012, 59(2):1571-1581.

24. Serrien DJ, Strens LH, Oliviero A, Brown P: Repetitive transcranial magnetic stimulation of the supplementary motor area (SMA) degrades bimanual movement control in humans. Neuroscience letters 2002, 328(2):89-92.

25. Halsband U, Matsuzaka Y, Tanji J: Neuronal activity in the primate supplementary, pre-supplementary and premotor cortex during externally and internally instructed sequential movements. Neuroscience 
research 1994, 20(2):149-155.

26. Koyama T, McHaffie JG, Laurienti PJ, Coghill RC: The subjective experience of pain: where expectations become reality. Proceedings of the National Academy of Sciences 2005, 102(36):12950-12955.

27. Hikosaka $\mathrm{O}$, Isoda M: Brain mechanisms for switching from automatic to controlled eye movements. In: Progress in brain research. Volume 171, edn.: Elsevier; 2008: 375-382.

28. Muhammad R, Wallis JD, Miller EK: A comparison of abstract rules in the prefrontal cortex, premotor cortex, inferior temporal cortex, and striatum. Journal of cognitive neuroscience 2006, 18(6):974-989.

29. Weinrich M, Wise S, Mauritz K-H: A neurophysiological study of the premotor cortex in the rhesus monkey. Brain 1984, 107(2):385-414.

30. Rizzolatti G, Scandolara C, Matelli M, Gentilucci M: Afferent properties of periarcuate neurons in macaque monkeys. II. Visual responses. Behavioural brain research 1981, 2(2):147-163.

31. Graziano MS, Reiss LA, Gross CG: A neuronal representation of the location of nearby sounds. Nature $1999,397(6718): 428$.

32. Fogassi L, Gallese V, Fadiga L, Luppino G, Matelli M, Rizzolatti G: Coding of peripersonal space in inferior premotor cortex (area F4). Journal of neurophysiology 1996, 76(1):141-157.

33. Wang M, Su J, Zhang J, Zhao Y, Yao Q, Zhang Q, Zhang H, Wang S, Li G-F, Liu J-R: Visual cortex and cerebellum hyperactivation during negative emotion picture stimuli in migraine patients. Scientific reports $2017,7: 41919$.

34. Yang Y, Song M, Fan Y, Ma K: Occipital nerve stimulation for migraine: a systematic review. Pain Practice 2016, 16(4):509-517.

35. Demarquay G, Royet J, Mick G, Ryvlin P: Olfactory hypersensitivity in migraineurs: a H2150-PET study. Cephalalgia 2008, 28(10):1069-1080.

36. Moulton E, Becerra L, Maleki N, Pendse G, Tully S, Hargreaves R, Burstein R, Borsook D: Painful heat reveals hyperexcitability of the temporal pole in interictal and ictal migraine states. Cerebral cortex 2010, 21(2):435-448.

37. Berlucchi G, Vallar G: The history of the neurophysiology and neurology of the parietal lobe. In: Handbook of clinical neurology. Volume 151, edn.: Elsevier; 2018: 3-30.

38. Wiech K, Ploner M, Tracey I: Neurocognitive aspects of pain perception. Trends in cognitive sciences 2008, 12(8):306-313.

39. Magon S, May A, Stankewitz A, Goadsby PJ, Schankin C, Ashina M, Amin FM, Seifert CL, Mallar Chakravarty M, Müller J: Cortical abnormalities in episodic migraine: A multi-center 3T MRI study. Cephalalgia 2019, 39(5):665-673.

40. Tomasi D, Volkow ND: Association between functional connectivity hubs and brain networks. Cerebral cortex 2011, 21(9):2003-2013.

41. Kim JH, Kim JB, Suh S-i, Seo W-K, Oh K, Koh S-B: Thickening of the somatosensory cortex in migraine without aura. Cephalalgia 2014, 34(14):1125-1133. 
42. Hu W, Guo J, Chen N, Guo J, He L: A meta-analysis of voxel-based morphometric studies on migraine. International journal of clinical and experimental medicine 2015, 8(3):4311.

43. Jia Z, Yu S: Grey matter alterations in migraine: a systematic review and meta-analysis. Neurolmage: Clinical 2017, 14:130-140.

44. Maihöfner $\mathrm{C}$, Handwerker HO, Birklein F: Functional imaging of allodynia in complex regional pain syndrome. Neurology 2006, 66(5):711-717.

45. Maihöfner $C$, Jesberger F, Seifert F, Kaltenhäuser M: Cortical processing of mechanical hyperalgesia: a MEG study. European Journal of Pain 2010, 14(1):64-70.

46. Burgmer M, Petzke F, Giesecke T, Gaubitz M, Heuft G, Pfleiderer B: Cerebral activation and catastrophizing during pain anticipation in patients with fibromyalgia. Psychosomatic medicine 2011, 73(9):751-759.

47. Zhang J, Wu Y-L, Su J, Yao Q, Wang M, Li G-F, Zhao R, Shi Y-H, Zhao Y, Zhang Q: Assessment of gray and white matter structural alterations in migraineurs without aura. The journal of headache and pain 2017, 18(1):74.

48. Qin Z, He X-W, Zhang J, Xu S, Li G-F, Su J, Shi Y-H, Ban S, Hu Y, Liu Y-S: Structural changes of cerebellum and brainstem in migraine without aura. The journal of headache and pain 2019, 20(1):93.

49. Moulton EA, Schmahmann JD, Becerra L, Borsook D: The cerebellum and pain: passive integrator or active participator? Brain research reviews 2010, 65(1):14-27.

50. Ruscheweyh R, Kühnel M, Filippopulos F, Blum B, Eggert T, Straube A: Altered experimental pain perception after cerebellar infarction. $P A I N \otimes 2014,155(7): 1303-1312$.

\section{Figures}


L M1

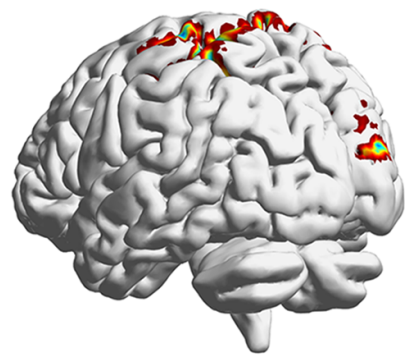

L

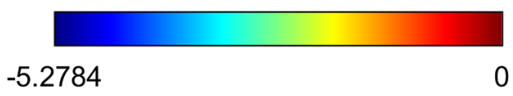

L S1

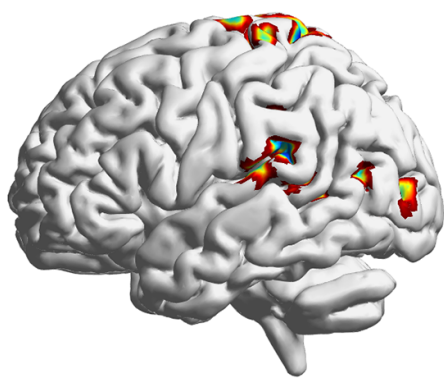

L

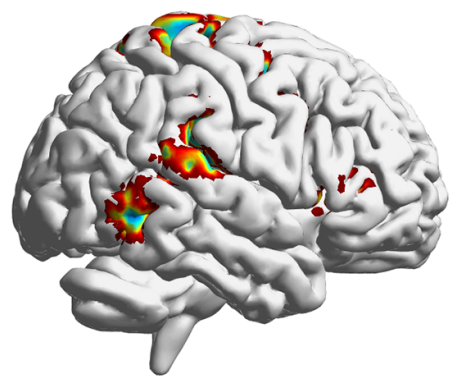

$\mathbf{R}$

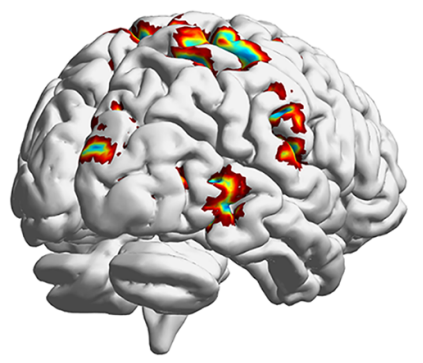

$\mathbf{R}$

R L

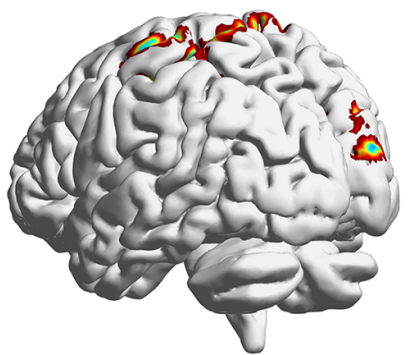

$-5.0019$
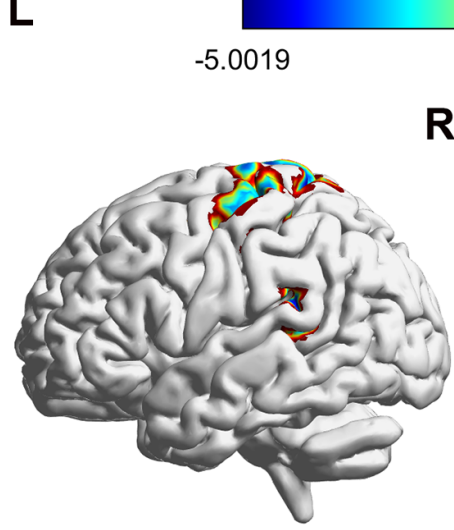

L
R M1

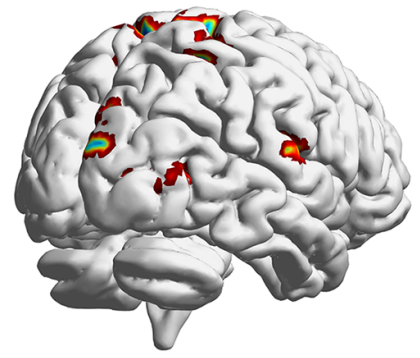

$\mathbf{R}$

R S1

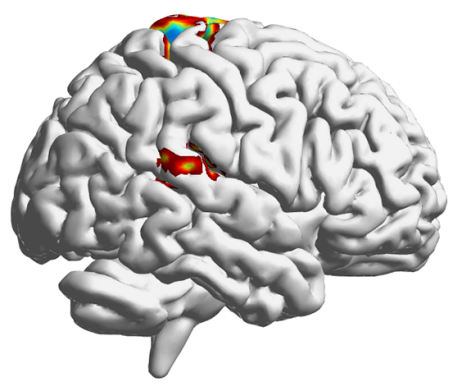

$\mathbf{R}$

$-5.7310$

0

$-5.1256$

0

Figure 1

Sagittal views of the MNI brain areas showing reduced intrinsic functional connectivity (FC) with the M1 and S1 in MWoAs compared to healthy controls. The surviving clusters were assigned thresholds at $p<0.001$ (voxel level) and were FWE corrected to $p<0.05$ at the cluster level. 
L SMA

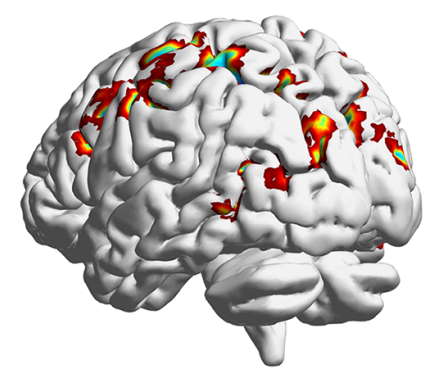

L

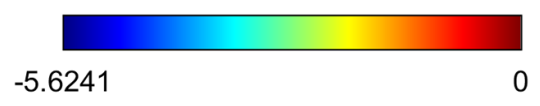

L PMv

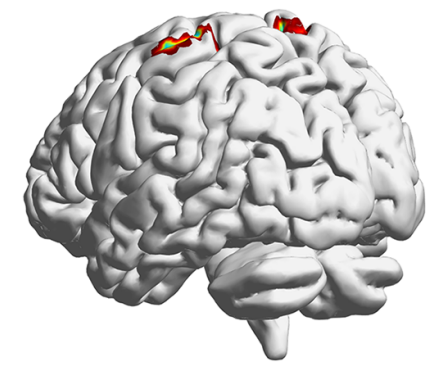

L

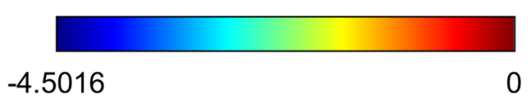

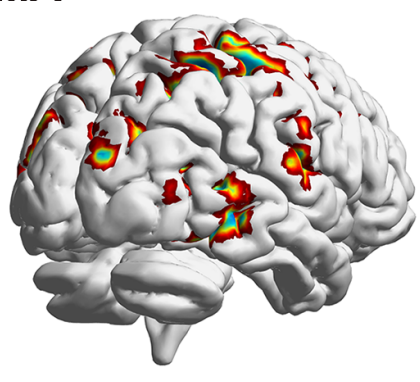

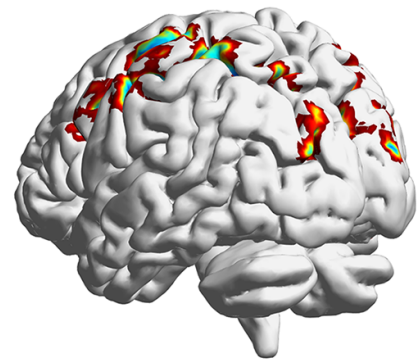

\section{R SMA}

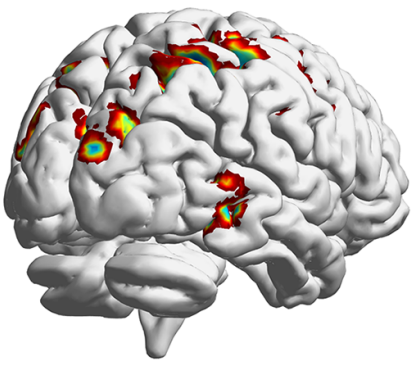

R L

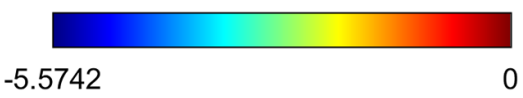

$\mathbf{R}$

R PMv
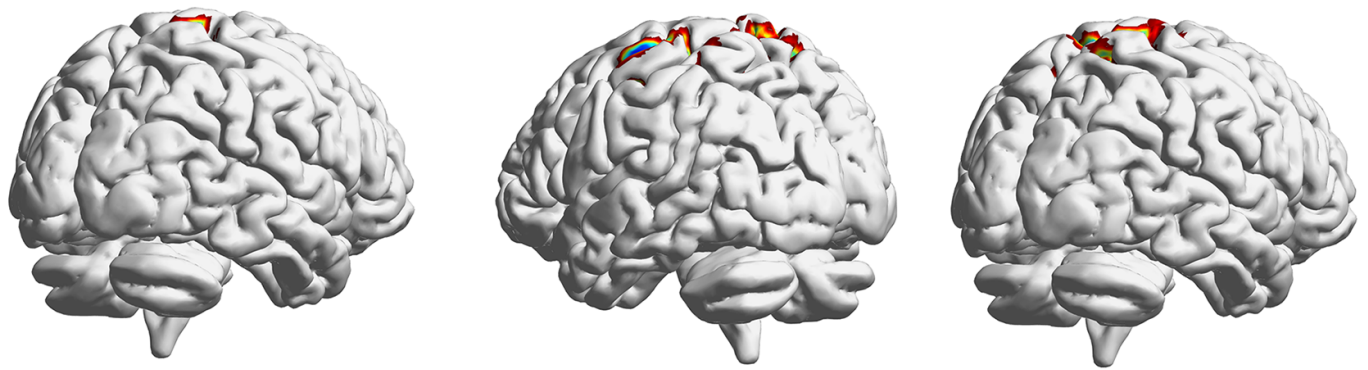

R L

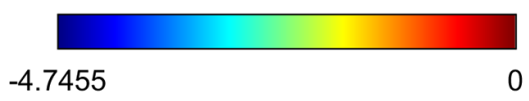

R

\section{Figure 2}

Sagittal views of the MNI brain areas showing reduced intrinsic functional connectivity (FC) with the SMA and PMv in MWoAs compared to healthy controls. The surviving clusters were assigned thresholds at $p<0.001$ (voxel level) and were FWE corrected to $p<0.05$ at the cluster level. 


\section{R pre-SMA}
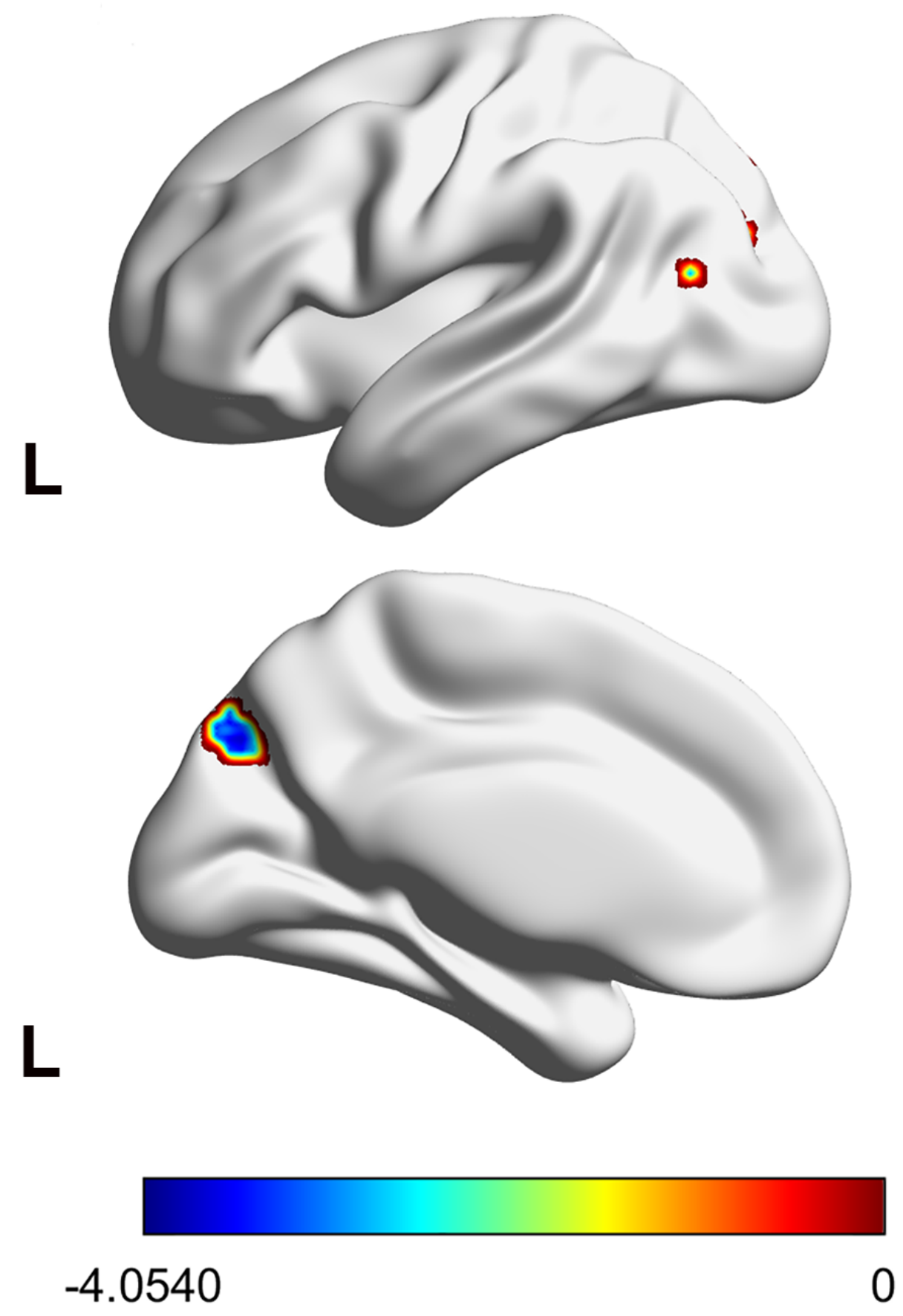

\section{Figure 3}

Sagittal views of the MNI brain areas showing reduced intrinsic functional connectivity (FC) with the preSMA in MWoAs compared to healthy controls. The surviving clusters were assigned thresholds at $p<0.001$ (voxel level) and were FWE corrected to $p<0.05$ at the cluster level. 


\section{R PMd}
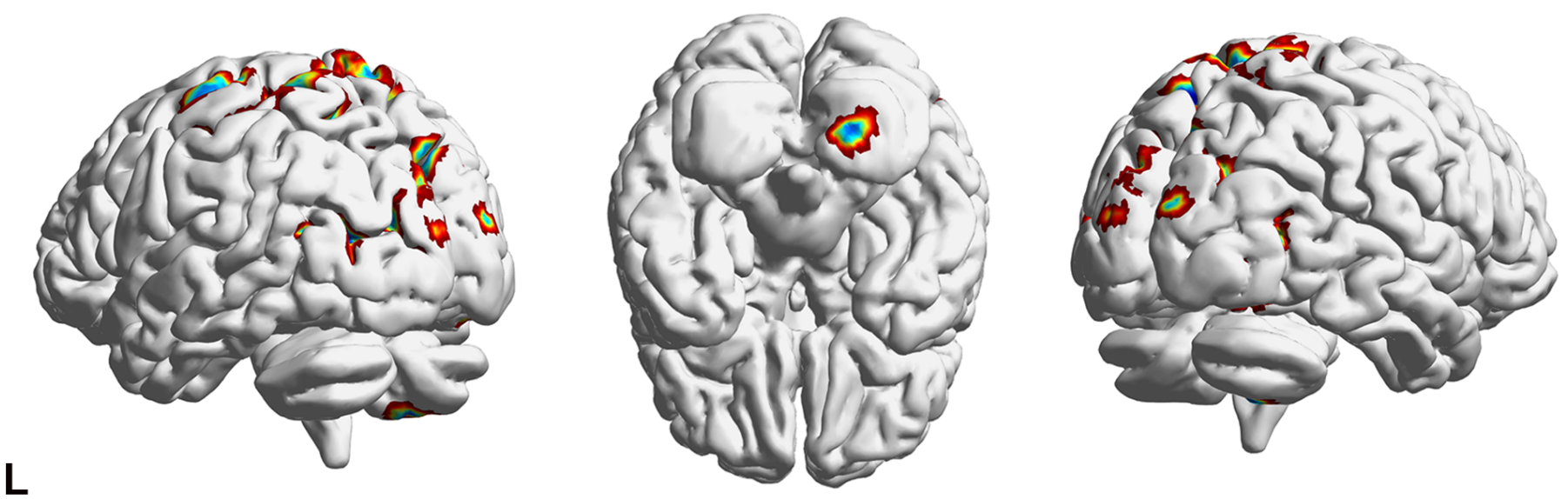

$\mathbf{R}$

$-4.8829$

Figure 4

Sagittal views of the MNI brain areas showing reduced intrinsic functional connectivity $(\mathrm{FC})$ with the PMd in MWoAs compared to healthy controls. The surviving clusters were assigned thresholds at $p<0.001$ (voxel level) and were FWE corrected to $p<0.05$ at the cluster level.

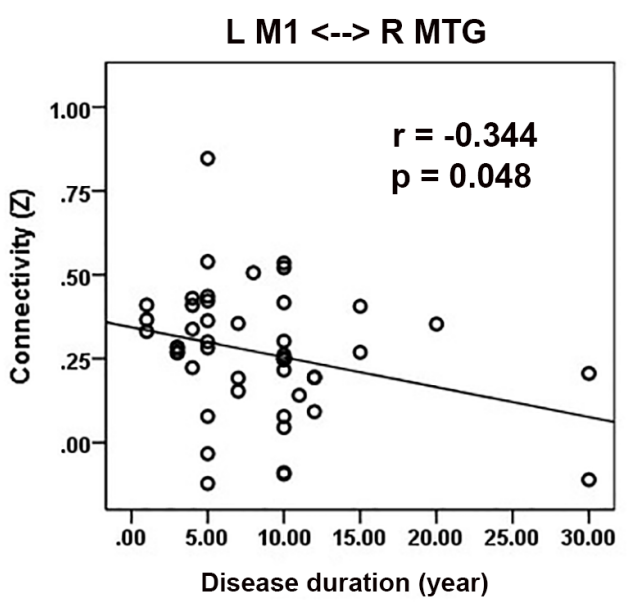

L S1 <--> R MTG

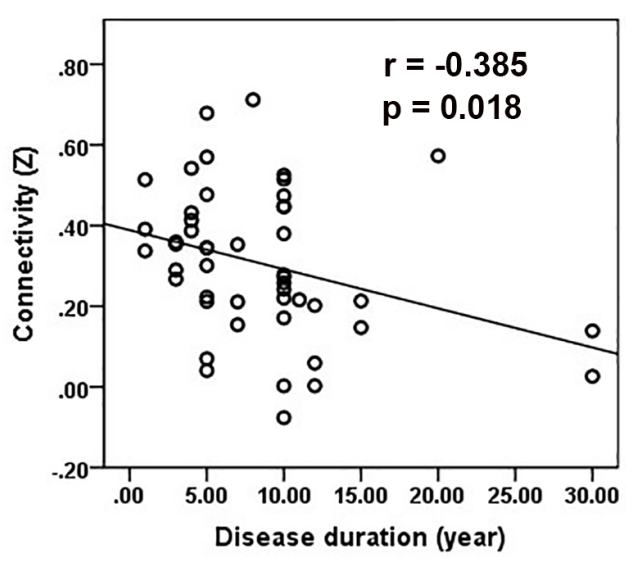

L M1 <--> R STG

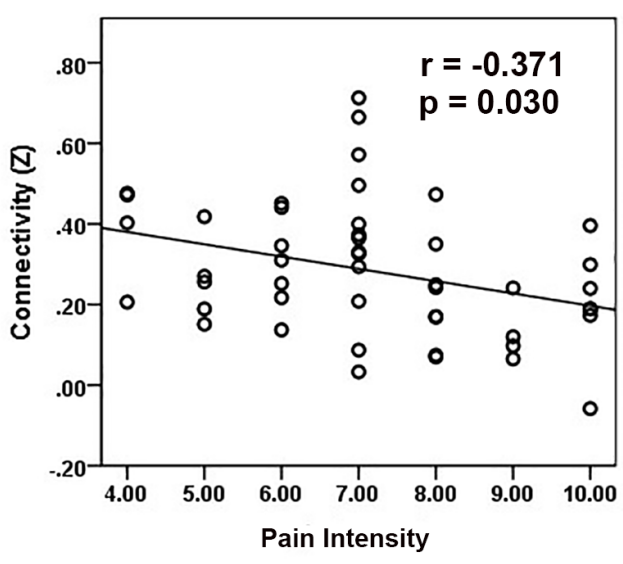

L S1 <--> R STG

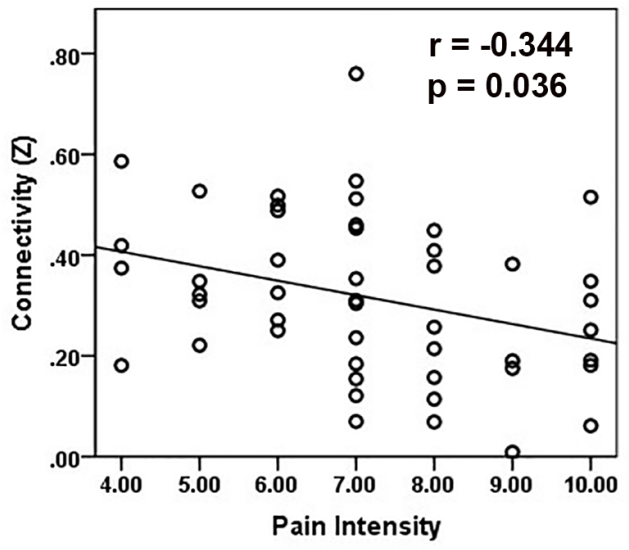

L PMV <--> R Cingulate gyrus

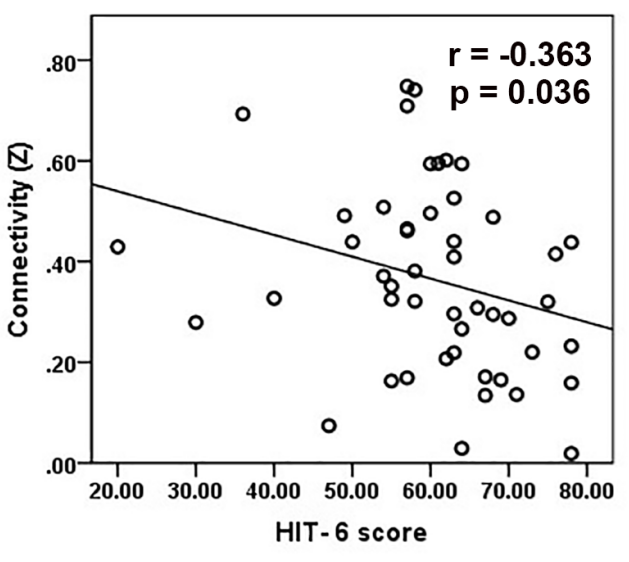

L S1 <--> R STG

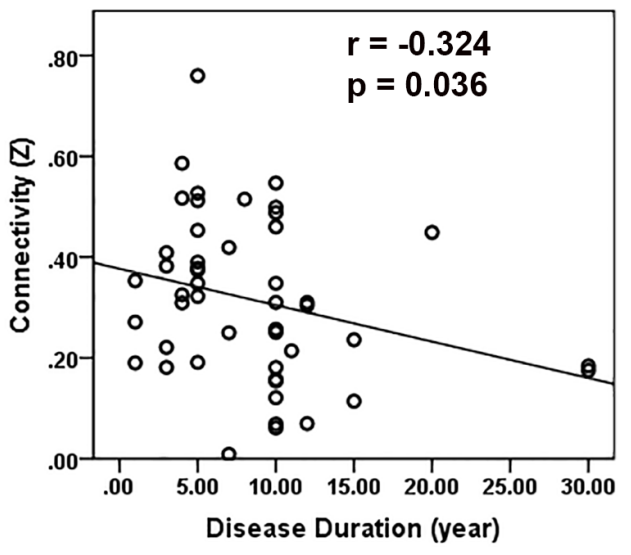




\section{Figure 5}

Scatter plot of clinical correlations of altered sensorimotor area functional connectivity (FC). Thresholds were $\mathrm{p}<0.05$, one-tailed, and FDR corrected for multiple comparisons. 